\title{
CRAMÉR TYPE LARGE DEVIATIONS FOR TRIMMED $L$-STATISTICS
}

\author{
BY
}

\author{
NADEZHDA GRIB KOVA* (St. Petersburg)
}

\begin{abstract}
In this paper, we propose a new approach to the investigation of asymptotic properties of trimmed $L$-statistics and we apply it to the Cramér type large deviation problem. Our results can be compared with those in Callaert et al. (1982) - the first and, as far as we know, the single article where some results on probabilities of large deviations for the trimmed $L$-statistics were obtained, but under some strict and unnatural conditions. Our approach is to approximate the trimmed $L$-statistic by a non-trimmed $L$-statistic (with smooth weight function) based on Winsorized random variables. Using this method, we establish the Cramér type large deviation results for the trimmed $L$-statistics under quite mild and natural conditions.
\end{abstract}

2010 AMS Mathematics Subject Classification: Primary: 62G30, 62E20; Secondary: 60F05, 60F10.

Key words and phrases: Trimmed $L$-statistics, central limit theorem, large deviations, moderate deviations.

\section{INTRODUCTION AND MAIN RESULTS}

Consider a sequence $X_{1}, X_{2}, \ldots$ of independent identically distributed realvalued random variables with distribution function $F$, and let $X_{1: n} \leqslant \ldots \leqslant X_{n: n}$ denote the order statistics corresponding to the first $n$ observations. Define the trimmed $L$-statistic by

$$
L_{n}=n^{-1} \sum_{i=k_{n}+1}^{n-m_{n}} c_{i, n} X_{i: n}
$$

where $c_{i, n} \in \mathbb{R}$, and $k_{n}, m_{n}$ are two sequences of integers such that $0 \leqslant k_{n}<$ $n-m_{n} \leqslant n$. Put $\alpha_{n}=k_{n} / n, \beta_{n}=m_{n} / n$. Throughout this paper, we suppose that $\alpha_{n} \rightarrow \alpha, \beta_{n} \rightarrow \beta$ as $n \rightarrow \infty$, where $0<\alpha<1-\beta<1$, i.e. we focus on the case of heavy trimmed $L$-statistic.

* Research partially supported by the Russian Foundation for Basic Research (grant RFBR no. SS-2504.2014.1). 
In this paper we investigate Cramér type large deviations, i.e. relative errors in the central limit theorem for $L_{n}$. First we note that in the case of non-trimmed $L$-statistic $\left(k_{n}=m_{n}=0\right)$ with coefficients $c_{i, n}$ generated by a smooth weight function the Cramér type large and moderate deviations were studied in a number of papers (see Vandemaele and Veraverbeke [29], Bentkus and Zitikis [3], Aleskeviciene [1]]). In contrast, to the best of our knowledge, there exists a sole paper Callaert et al. [6] - devoted to the large deviations for the trimmed $L$-statistics. However, the result in [6] was obtained under some rigorous and unnatural conditions imposed on the underlying distribution $F$ and the weights. The method of proof in Callaert et al. [6] is based on the following two well-known facts:

1. The joint distribution of $X_{i: n}$ coincides with the joint distribution of $F^{-1}\left(G\left(Z_{i: n}\right)\right), i=1, \ldots, n$, where $G$ is the distribution function of the standard exponential distribution, $Z_{i: n}$ are the order statistics corresponding to a sample of $n$ independent random variables from the distribution $G$.

2. The order statistics $Z_{i: n}$ are distributed as $\sum_{k=1}^{i} Z_{k} /(n-k+1)$, where $Z_{k}$ are independent standard exponential random variables.

These two facts and the Taylor expansion together enable one to get an approximation of $L_{n}$ by a sum of weighted i.i.d. random variables for which some suitable known result on Cramér type large deviations can be applied. This approach was first implemented by Bjerve [4] to prove a Berry-Esseen type result for the $L$-statistics. However, the use of this method requires excessive smoothness conditions imposed on $F$ and leads to the unnatural and complicated normalization of the $L$-statistic (cf. Callaert et al. [6]).

In this article, we propose another approach to the investigation of asymptotic properties of the trimmed $L$-statistics different from that used in Bjerve [4] and Callaert et al. [6]. Our idea is to approximate the trimmed $L$-statistic by a nontrimmed $L$-statistic with weights generated by a smooth weight function, where the approximating $L$-statistic is based on the order statistics corresponding to a sample of $n$ i.i.d. Winsorized random variables. The asymptotic properties that we are interested in are often well studied in the case of $L$-statistics with a smooth weight function and bounded observations. This allows us to obtain a desired result for the trimmed $L$-statistic by applying a result of the corresponding type to the approximating non-trimmed $L$-statistic; so it remains only to evaluate the remainder in the approximation. Here, we apply our method to obtain a result on probabilities of large deviations for the trimmed $L$-statistics, and we establish it under mild and natural conditions. Our result on large deviations can be viewed as a strengthening of the result from Callaert et al. [6].

To conclude this introduction, we adduce a brief review of the relevant literature. The class of $L$-statistics is one of the most commonly used classes in statistical inferences. We refer to monographs by David and Nagaraja [7], Serfling [23], Shorack and Wellner [26], van der Vaart [30] for an introduction to the theory and applications of $L$-statistics. There is a vast literature on asymptotic properties of $L$ statistics. Since we focus on the case of heavy trimmed $L$-statistics, we will men- 
tion mainly sources appropriate to our case. The most significant contribution to the establishment of the central limit theorem for (trimmed) $L$-statistics was made by Shorack [24], [25], and Stigler [27], [28]. Mason and Shorack [27] obtained the necessary and sufficient conditions for the asymptotic normality of the trimmed $L$-statistics. The Berry-Esseen type bounds under different sets of conditions were obtained by Bjerve [4], Helmers [18], [19], Gribkova [11]. A great contribution to the research of second order asymptotic properties for $L$-statistic was done by Helmers [16]-[19], who established the Edgeworth expansions for the (trimmed) $L$-statistics. In papers by Bentkus et al. [2], Friedrich [8], Putter and van Zwet [22] and van Zwet [31], the Berry-Esseen type bounds and Edgeworth expansions for $L$-statistics were derived as the consequences of the very general results for symmetric statistics established in these papers. Some interesting results on Chernoff's type large deviations (for non-trimmed $L$-statistics with smooth weight function) were obtained by Boistard [5]. Recently, Gao and Zhao [9] proposed a general delta method in the theory of Chernoff's type large deviations and illustrated it by many examples including $M$-estimators and $L$-statistics. A survey on the $L$-statistics and some modern applications of them in the economy and theory of actuarial risks can be found in Greselin et al. [10].

We will now proceed to the statement of our results. Define the left-continuous inverse of $F: F^{-1}(u)=\inf \{x: F(x) \geqslant u\}, 0<u \leqslant 1, F^{-1}(0)=F^{-1}\left(0^{+}\right)$, and let $F_{n}, F_{n}^{-1}$ denote the empirical distribution function and its inverse, respectively. Let $J$ be a function defined in an open set $I$ such that $[\alpha, 1-\beta] \subset I \subseteq(0,1)$. We will also consider the trimmed $L$-statistics with coefficients generated by the weight function $J$,

$$
L_{n}^{0}=n^{-1} \sum_{i=k_{n}+1}^{n-m_{n}} c_{i, n}^{0} X_{i: n}=\int_{\alpha_{n}}^{1-\beta_{n}} J(u) F_{n}^{-1}(u) d u,
$$

where $c_{i, n}^{0}=n \int_{(i-1) / n}^{i / n} J(u) d u$.

To state our results, we will need the following set of assumptions.

(i) $J$ is Lipschitz in $I$, i.e. there exists a constant $C \geqslant 0$ such that

$$
|J(u)-J(v)| \leqslant C|u-v| \quad \text { for all } u, v \in I .
$$

(ii) $F^{-1}$ satisfies a Hölder condition of order $0<\varepsilon \leqslant 1$ in some neighborhoods $U_{\alpha}$ and $U_{1-\beta}$ of $\alpha$ and $1-\beta$.

(iii) $\max \left(\left|\alpha_{n}-\alpha\right|,\left|\beta_{n}-\beta\right|\right)=O\left(n^{-1 /(2+\varepsilon)}\right)$, where $\varepsilon$ is the Hölder index from condition (ii).

(iv) With $\varepsilon$ from conditions (ii) and (iii)

$$
\sum_{i=k_{n}+1}^{n-m_{n}}\left|c_{i, n}-c_{i, n}^{0}\right|=O\left(n^{1 /(2+\varepsilon)}\right) .
$$


Define a sequence of centering constants

$$
\mu_{n}=\int_{\alpha_{n}}^{1-\beta_{n}} J(u) F^{-1}(u) d u .
$$

Since $\alpha_{n} \rightarrow \alpha, \beta_{n} \rightarrow \beta$ as $n \rightarrow \infty$, both variables $L_{n}^{0}$ and $\mu_{n}$ are well defined for all sufficiently large $n$.

It is well known (cf., e.g., [21], [28], [30]) that when the inverse $F^{-1}$ is continuous at two points $\alpha$ and $1-\beta$, the smoothness condition (ㄴ.3) implies the weak convergence to the normal law: $\sqrt{n}\left(L_{n}^{0}-\mu_{n}\right) \Rightarrow N\left(0, \sigma^{2}\right)$, where

$$
\sigma^{2}=\sigma^{2}(J, F)=\int_{\alpha}^{1-\beta} \int_{\alpha}^{1-\beta} J(u) J(v)(u \wedge v-u v) d F^{-1}(u) d F^{-1}(v),
$$

and $u \wedge v=\min (u, v)$. Here and in the sequel, we use the convention that $\int_{a}^{b}=$ $\int_{[a, b)}$ when integrating with respect to the left continuous integrator $F^{-1}$. All along the article, we assume $\sigma>0$.

Define the distribution functions of the normalized $L_{n}$ and $L_{n}^{0}$, respectively:

$$
F_{L_{n}}(x)=\mathbf{P}\left\{\sqrt{n}\left(L_{n}-\mu_{n}\right) / \sigma \leqslant x\right\}, \quad F_{L_{n}^{0}}(x)=\mathbf{P}\left\{\sqrt{n}\left(L_{n}^{0}-\mu_{n}\right) / \sigma \leqslant x\right\} .
$$

Let $\Phi$ denote the standard normal distribution function. Here is our first result on Cramér type large deviations for $L_{n}$.

THEOREM 1.1. Suppose that $F^{-1}$ satisfies condition (ii) for some $0<\varepsilon \leqslant 1$, and the sequences $\alpha_{n}$ and $\beta_{n}$ satisfy (iii). In addition, assume that the weights $c_{i, n}$ satisfy (iv) for some function $J$ satisfying condition (i).

Then for every sequence $a_{n} \rightarrow 0$ and each $A>0$

$$
\begin{aligned}
1-F_{L_{n}}(x) & =[1-\Phi(x)](1+o(1)), \\
F_{L_{n}}(-x) & =\Phi(-x)(1+o(1)),
\end{aligned}
$$

as $n \rightarrow \infty$, uniformly in the range $-A \leqslant x \leqslant a_{n} n^{\varepsilon /(2(2+\varepsilon))}$.

The proof of our main results is relegated to Section 3 . Theorem $\mathbb{L}$ d directly implies the following two corollaries.

COROLLARY 1.1. Suppose that the conditions of Theorem $\mathbb{\square}$ are satisfied with $\varepsilon=1$, i.e. $F^{-1}$ is Lipschitz in some neighborhoods $U_{\alpha}$ and $U_{1-\beta}$ of $\alpha$ and $1-\beta$. Then for every sequence $a_{n} \rightarrow 0$ and each $A>0$ the relations (IL.7) hold true, uniformly in the range $-A \leqslant x \leqslant a_{n} n^{1 / 6}$.

COROLlaRY 1.2. Let $c_{i, n}=c_{i, n}^{0}=n \int_{(i-1) / n}^{i / n} J(u) d u, k_{n}+1 \leqslant i \leqslant n-m_{n}$, where $J$ is a function satisfying (i). Furthermore, assume that conditions (ii) and 
(iii) hold for some $0<\varepsilon \leqslant 1$. Then the relations (1.7) with $L_{n}=L_{n}^{0}$ hold true for every sequence $a_{n} \rightarrow 0$ and each $A>0$, uniformly in the range $-A \leqslant x \leqslant$ $a_{n} n^{\varepsilon /(2(2+\varepsilon))}$.

Theorem $\square$.$] can be compared with the result by Callaert et al. [6], where$ it was assumed that the derivative $H^{\prime}=\left(F^{-1} \circ G\right)^{\prime}$ exists and satisfies a Hölder condition of order $0<\varepsilon \leqslant 1$ in some open set containing $\left[G^{-1}(\alpha), G^{-1}(1-\beta)\right]$, where $G$ is the standard exponential distribution function. Moreover, some unnatural condition was imposed on the weights and $H^{\prime}$ (cf. conditions (A2) and (B) in Callaert et al. [6]). In contrast, we use the natural scale parameter $\sigma-$ the root of the asymptotic variance of $L_{n}$ - for the normalization, and our smoothness condition (ii) for $F^{-1}$ is much weaker than that from Callaert et al. [6].

Our Theorem $\square$ is also related to previous results by Vandemaele and Veraverbeke [29] and Bentkus and Zitikis [3] on Cramér type large deviations for nontrimmed $L$-statistics with smooth weight function. The method of proof in the first of these articles was based on Helmers's ([18], [19]) $U$-statistic approximation, and in the second one the $\omega^{2}$-von Mises statistic type approximation was applied. We approximate our trimmed $L$-statistic by $L$-statistics with smooth weight function. Moreover, we apply the results from the papers mentioned to our approximating non-trimmed $L$-statistic when proving Theorem I.1. Note also that Cramér's moment conditions for the underlying distribution assumed in the cited papers are not needed in the case of the trimmed $L$-statistics, whereas the smoothness of $F^{-1}$ near $\alpha$ and $1-\beta$ becomes essential for the Cramér type large deviation results.

Finally, we state a version of Theorem $\square$.1, where the scale factor $\sigma / n^{1 / 2}$ is replaced by $\sqrt{\operatorname{Var}\left(L_{n}\right)}$, it is parallel to Theorem 2 (ii) by Vandemaele and Veraverbeke [29], but now for the trimmed $L$-statistics.

We will need the following two somewhat stronger versions of conditions (iii) and (iv):

(iii') $\max \left(\left|\alpha_{n}-\alpha\right|,\left|\beta_{n}-\beta\right|\right)=O\left(n^{-\frac{1}{2+\varepsilon}\left[1+\frac{\varepsilon(1-\varepsilon)}{2}\right]}(\log n)^{-\varepsilon / 2}\right)$, where $\varepsilon$ is the Hölder index from condition (ii).

$\left(\mathrm{iv}^{\prime}\right)$ With $\varepsilon$ from conditions (ii) and (iii')

$$
\sum_{i=k_{n}+1}^{n-m_{n}}\left|c_{i, n}-c_{i, n}^{0}\right|=O\left(n^{\frac{1}{2+\varepsilon}\left[1-\frac{\varepsilon}{2}\right]}\right) .
$$

THEOREM 1.2. Suppose that the conditions of Theorem $\square$.$] are satisfied,$ where (iii) and (iv) are replaced by (iii') and (iv'), respectively. In addition, assume that $\operatorname{Var}\left(L_{n}\right)<\infty$ for all sufficiently large $n$. Then

$$
n \sigma^{-2} \operatorname{Var}\left(L_{n}\right)=1+O\left(n^{-\varepsilon /(2+\varepsilon)}\right) .
$$

Furthermore, the relations (․ㄱ), where $\sigma / n^{1 / 2}$ is replaced by $\sqrt{\operatorname{Var}\left(L_{n}\right)}$, hold true for every sequence $a_{n} \rightarrow 0$ and each $A>0$ as $n \rightarrow \infty$, uniformly in the range $-A \leqslant x \leqslant a_{n} n^{\varepsilon /(2(2+\varepsilon))}$. 
Note that in the case of heavy trimmed $L$-statistics the condition $\mathbf{E}\left|X_{1}\right|^{\gamma}<\infty$ (for some $\gamma>0$ ) is sufficient for the finiteness of $\operatorname{Var}\left(L_{n}\right)$ when $n$ gets large.

\section{OUR METHOD (REPRESENTATION FOR $L_{N}^{0}$ BY A NON-TRIMMED $L$-STATISTIC)}

Let $\xi_{\nu}=F^{-1}(\nu), 0<\nu<1$, be the $\nu$-th quantile of $F$, and $W_{i}$ denote $X_{i}$ Winsorized outside of $\left(\xi_{\alpha}, \xi_{1-\beta}\right]$. In other words,

$$
W_{i}= \begin{cases}\xi_{\alpha}, & X_{i} \leqslant \xi_{\alpha}, \\ X_{i}, & \xi_{\alpha}<X_{i} \leqslant \xi_{1-\beta}, \\ \xi_{1-\beta}, & \xi_{1-\beta}<X_{i} .\end{cases}
$$

Let $W_{i: n}$ denote the order statistics corresponding to $W_{1}, \ldots, W_{n}$ (the sample of $n$ i.i.d. auxiliary random variables).

Define the distribution function $G(x)=\mathbf{P}\left\{W_{i} \leqslant x\right\}$ of $W_{i}$, the corresponding quantile function is equal to $G^{-1}(u)=\xi_{\alpha} \vee\left(F^{-1}(u) \wedge \xi_{1-\beta}\right)$. Here and further on, $(a \vee b)=\max (a, b)$. Let $G_{n}$ and $G_{n}^{-1}$ denote the corresponding empirical distribution function and its inversion, respectively.

We will approximate $L_{n}$ by a linear combination of the order statistics $W_{i: n}$ with coefficients generated by the weight function

$$
J_{w}(u)= \begin{cases}J(\alpha), & u \leqslant \alpha, \\ J(u), & \alpha<u \leqslant 1-\beta, \\ J(1-\beta), & 1-\beta<u,\end{cases}
$$

which is defined in $[0,1]$. It is obvious that when $J$ is Lipschitz in $I$, i.e. satisfies condition (ㄴ.3) with some positive constant $C$, the function $J_{w}$ is Lipschitz in $[0,1]$ with some constant $C_{w} \leqslant C$.

Consider the auxiliary non-truncated $L$-statistic given by

$$
\widetilde{L}_{n}=n^{-1} \sum_{i=1}^{n} \widetilde{c}_{i, n} W_{i: n}=\int_{0}^{1} J_{w}(u) G_{n}^{-1}(u) d u,
$$

where $\widetilde{c}_{i, n}=n \int_{(i-1) / n}^{i / n} J_{w}(u) d u$. Define the centering constants

$$
\mu_{\widetilde{L}_{n}}=\int_{0}^{1} J_{w}(u) G^{-1}(u) d u
$$

Since $W_{i}$ has the finite moments of any order and because $J_{w}$ is Lipschitz, the distribution of the normalized $\widetilde{L}_{n}$ tends to the standard normal law (see, e.g., [28]),

$$
\sqrt{n}\left(\widetilde{L}_{n}-\mu_{\widetilde{L}_{n}}\right) / \sigma\left(J_{w}, G\right) \Rightarrow N(0,1),
$$


where the asymptotic variance

$$
\sigma^{2}\left(J_{w}, G\right)=\int_{0}^{1} \int_{0}^{1} J_{w}(u) J_{w}(v)(u \wedge v-u v) d G^{-1}(u) d G^{-1}(v) .
$$

Observe that for $u \in(\alpha, 1-\beta]$ we have $J_{w}(u)=J(u), G^{-1}(u)=F^{-1}(u)$, and that $d G^{-1}(u) \equiv 0$ for $u \notin(\alpha, 1-\beta]$. This yields the equality of the asymptotic variances

$$
\sigma^{2}\left(J_{w}, G\right)=\sigma^{2}(J, F)=\sigma^{2}
$$

of the truncated $L$-statistic $L_{n}^{0}$ and the non-truncated $L$-statistic $\widetilde{L}_{n}$ based on the Winsorized random variables.

Define the binomial random variable $N_{\nu}=\sharp\left\{i: X_{i} \leqslant \xi_{\nu}\right\}$, where $0<\nu<1$. Our representation for $L_{n}^{0}$ is based on the following simple observation: we see that

$$
W_{i: n}= \begin{cases}\xi_{\alpha}, & i \leqslant N_{\alpha} \\ X_{i: n}, & N_{\alpha}<i \leqslant N_{1-\beta} \\ \xi_{1-\beta}, & i>N_{1-\beta}\end{cases}
$$

Put $A_{n}=N_{\alpha} / n$ and $B_{n}=\left(n-N_{1-\beta}\right) / n$. The following lemma provides us a useful representation which is crucial in the proof of our main results.

LEMMA 2.1. We have

$$
L_{n}^{0}-\mu_{n}=\widetilde{L}_{n}-\mu_{\widetilde{L}_{n}}+R_{n}
$$

where $R_{n}=R_{n}^{(1)}+R_{n}^{(2)}$,

$$
R_{n}^{(1)}=\int_{\alpha}^{A_{n}} J_{w}(u)\left[F_{n}^{-1}(u)-\xi_{\alpha}\right] d u-\int_{1-\beta}^{1-B_{n}} J_{w}(u)\left[F_{n}^{-1}(u)-\xi_{1-\beta}\right] d u
$$

and

$$
R_{n}^{(2)}=\int_{\alpha_{n}}^{\alpha} J(u)\left[F_{n}^{-1}(u)-F^{-1}(u)\right] d u-\int_{1-\beta_{n}}^{1-\beta} J(u)\left[F_{n}^{-1}(u)-F^{-1}(u)\right] d u .
$$

Pro of. First, consider the difference between the centering constants. We obtain

$$
\begin{aligned}
& \text { (2.11) } \mu_{\widetilde{L}_{n}}-\mu_{n}=\int_{0}^{1} J_{w}(u) G^{-1}(u) d u-\int_{\alpha_{n}}^{1-\beta_{n}} J(u) F^{-1}(u) d u \\
& =\alpha J(\alpha) \xi_{\alpha}+\beta J(1-\beta) \xi_{1-\beta}-\int_{\alpha_{n}}^{\alpha} J(u) F^{-1}(u) d u+\int_{1-\beta_{n}}^{1-\beta} J(u) F^{-1}(u) d u .
\end{aligned}
$$


For the difference between $L_{n}^{0}$ and $\widetilde{L}_{n}$, after some simple computations, we get

$$
\begin{aligned}
L_{n}^{0}-\widetilde{L}_{n}= & \int_{\alpha}^{1-\beta} J(u)\left[F_{n}^{-1}(u)-G_{n}^{-1}(u)\right] d u \\
& +\int_{\alpha_{n}}^{\alpha} J(u) F_{n}^{-1}(u) d u-\int_{1-\beta_{n}}^{1-\beta} J(u) F_{n}^{-1}(u) d u \\
& -J(\alpha) \int_{0}^{\alpha} G_{n}^{-1}(u) d u-J(1-\beta) \int_{1-\beta}^{1} G_{n}^{-1}(u) d u .
\end{aligned}
$$

The relations (2.JI) and (2.12) together imply

$$
L_{n}^{0}-\widetilde{L}_{n}+\left(\mu_{\widetilde{L}_{n}}-\mu_{n}\right)=D_{n}+R_{n}^{(2)},
$$

where

$$
\begin{aligned}
D_{n}:= & \int_{\alpha}^{1-\beta} J(u)\left[F_{n}^{-1}(u)-G_{n}^{-1}(u)\right] d u \\
& +J(\alpha)\left[\alpha \xi_{\alpha}-\int_{0}^{\alpha} G_{n}^{-1}(u) d u\right]+J(1-\beta)\left[\beta \xi_{1-\beta}-\int_{1-\beta}^{1} G_{n}^{-1}(u) d u\right] .
\end{aligned}
$$

It remains to show that $D_{n}=R_{n}^{(1)}$. Let us consider three of six possible cases (treatment for the three other cases is similar, and therefore omitted). We use the fact that $F_{n}^{-1}(u)=G_{n}^{-1}(u)$ for $A_{n}<u \leqslant 1-B_{n}, G_{n}^{-1}(u)=\xi_{\alpha}$ for $u \leqslant A_{n}$, and $G_{n}^{-1}(u)=\xi_{1-\beta}$ for $u>1-B_{n}$.

C a s e $1 . \alpha \leqslant A_{n} \leqslant 1-B_{n}<1-\beta$. In this case the second and third terms of $D_{n}$ are equal to zero, and the first one yields

$$
D_{n}=\int_{\alpha}^{A_{n}} J(u)\left[F_{n}^{-1}(u)-\xi_{\alpha}\right] d u+\int_{1-B_{n}}^{1-\beta} J(u)\left[F_{n}^{-1}(u)-\xi_{1-\beta}\right] d u
$$

and since $J(u)=J_{w}(u)$ for $\alpha<u \leqslant 1-\beta$, we obtain the desired equality.

Ca se $2 . \alpha \leqslant A_{n} \leqslant 1-\beta<1-B_{n}$. In this case we have

$$
\begin{aligned}
D_{n}= & \int_{\alpha}^{A_{n}} J(u)\left[F_{n}^{-1}(u)-\xi_{\alpha}\right] d u \\
& +J(1-\beta)\left[\beta \xi_{1-\beta}-\int_{1-\beta}^{1-B_{n}} F_{n}^{-1}(u) d u-B_{n} \xi_{1-\beta}\right] \\
= & \int_{\alpha}^{A_{n}} J(u)\left[F_{n}^{-1}(u)-\xi_{\alpha}\right] d u-\int_{1-\beta}^{1-B_{n}} J(1-\beta)\left[F_{n}^{-1}(u)-\xi_{1-\beta}\right] d u,
\end{aligned}
$$


and since $J(u)=J_{w}(u)$ for $\alpha<u \leqslant A_{n}$ and $J(1-\beta)=J_{w}(u)$ for $u>1-\beta$, the expression on the right-hand side in (2.14) is equal to $R_{n}^{(1)}$.

Cas e $3.1-\beta \leqslant A_{n} \leqslant 1-B_{n}$. In this case $D_{n}$ can be written as

$$
\begin{aligned}
& \text { (2.16) } \int_{\alpha}^{A_{n}} J_{w}(u)\left[F_{n}^{-1}(u)-\xi_{\alpha}\right] d u \\
& -J(1-\beta) \int_{1-\beta}^{A_{n}} F_{n}^{-1}(u) d u+J(1-\beta) \xi_{\alpha}\left(A_{n}-(1-\beta)\right) \\
& +J(1-\beta)\left[\beta \xi_{1-\beta}-\xi_{\alpha}\left(A_{n}-(1-\beta)\right)-\int_{A_{n}}^{1-B_{n}} F_{n}^{-1}(u) d u-B_{n} \xi_{1-\beta}\right] \\
& =\int_{\alpha}^{A_{n}} J_{w}(u)\left[F_{n}^{-1}(u)-\xi_{\alpha}\right] d u-\int_{1-\beta}^{1-B_{n}} J(1-\beta)\left[F_{n}^{-1}(u)-\xi_{1-\beta}\right] d u=R_{n}^{(1)} .
\end{aligned}
$$

This completes the proof of the representation (2.8). The lemma is proved.

In conclusion of this section, we note that the idea of the $L$-statistic approximation emerged as a result of the observation of the fact that the asymptotic variances of $L_{n}^{0}$ and of the non-trimmed $L$-statistic $\widetilde{L}_{n}$ based on the Winsorized random variables coincide. This idea of the $L$-statistic approximation can also be regarded as an extension of the one used in Gribkova and Helmers [12], [13] and [15] (where the second order asymptotic properties - the Berry-Esseen bounds and Edgeworth type expansions - were established for (slightly) trimmed means and their Studentized versions) to the case of trimmed $L$-statistics. In the papers mentioned, we constructed the $U$-statistic type approximations for (slightly) trimmed means using sums of i.i.d. Winsorized observations as the linear $U$-statistic terms; in order to get the quadratic terms, we applied some special Bahadur-Kiefer representations of von Mises statistic type for (intermediate) sample quantiles (cf. Gribkova and Helmers [14]).

\section{PROOF OF THEOREMS 1.1 AND 1.2}

Pro of of The or e m ㄷ. l. Obviously, it suffices to prove the first of the relations (1L.7). Set

$$
V_{n}=L_{n}-L_{n}^{0}=n^{-1} \sum_{i=k_{n}+1}^{n-m_{n}}\left(c_{i, n}-c_{i, n}^{0}\right) X_{i: n}
$$

Lemma 2.$]$ and the relation (B.]) together yield

$$
L_{n}-\mu_{n}=\widetilde{L}_{n}-\mu_{\widetilde{L}_{n}}+R_{n}+V_{n}
$$


In view of the classical Slutsky argument applied to (B.2), $1-F_{L_{n}}(x)$ is bounded above and below by

$$
\mathbf{P}\left\{\sqrt{n}\left(\widetilde{L}_{n}-\mu_{\widetilde{L}_{n}}\right) / \sigma>x-2 \delta\right\}+\mathbf{P}\left\{\sqrt{n}\left|R_{n}\right| / \sigma>\delta\right\}+\mathbf{P}\left\{\sqrt{n}\left|V_{n}\right| / \sigma>\delta\right\}
$$

and

$$
\mathbf{P}\left\{\sqrt{n}\left(\widetilde{L}_{n}-\mu_{\widetilde{L}_{n}}\right) / \sigma>x+2 \delta\right\}-\mathbf{P}\left\{\sqrt{n}\left|R_{n}\right| / \sigma>\delta\right\}-\mathbf{P}\left\{\sqrt{n}\left|V_{n}\right| / \sigma>\delta\right\},
$$

respectively, for each $\delta>0$.

Let $z_{n}=n^{\varepsilon /(2(2+\varepsilon))}$. Fix an arbitrary sequence $a_{n} \rightarrow 0$ and $A>0$. Without loss of generality we may assume that $a_{n} \geqslant 1 / \log (1+n)$ (otherwise, we may replace $a_{n}$ by the new sequence $a_{n}^{\prime}=\max \left(a_{n}, 1 / \log (1+n)\right) \geqslant a_{n}$ without affecting result). Set $\delta=\delta_{n}=a_{n}^{-1 / 2} / z_{n}$. From (B.3) and (3.4) it follows immediately that to prove our theorem it suffices to show that

$$
\begin{gathered}
\mathbf{P}\left\{\sqrt{n}\left(\widetilde{L}_{n}-\mu_{\widetilde{L}_{n}}\right) / \sigma>x \pm 2 \delta\right\}=[1-\Phi(x)](1+o(1)), \\
\mathbf{P}\left\{\sqrt{n}\left|R_{n}\right| / \sigma>\delta\right\}=[1-\Phi(x)] o(1), \\
\mathbf{P}\left\{\sqrt{n}\left|V_{n}\right| / \sigma>\delta\right\}=[1-\Phi(x)] o(1),
\end{gathered}
$$

uniformly in the range $-A \leqslant x \leqslant a_{n} z_{n}$.

Pr o of of (3.5). Since $\widetilde{L}_{n}$ is the non-truncated linear combination of order statistics corresponding to the sample $W_{1}, \ldots, W_{n}$ of i.i.d. bounded random variables and because its weight function $J_{w}$ is Lipschitz in $[0,1]$, we can apply the results on probabilities of large deviations by Vandemaele and Veraverbeke [29] and by Bentkus and Zitikis [3]. Set $B=A+2 \sup _{n \geqslant 1} \delta_{n}$ and $b_{n}=a_{n}+2 \delta_{n}$. Since $a_{n} \geqslant 1 / \log (1+n)$, the number $B$ exists, and $b_{n} \rightarrow 0$. Then, by Theorem 2 (i) of Vandemaele and Veraverbeke [29] for $x$ such that $-B \leqslant x \pm 2 \delta<0$, and by Theorem 1.1 of Bentkus and Zitikis [3] for $x$ such that $0 \leqslant x \pm 2 \delta \leqslant b_{n} n^{1 / 6}$,

$$
\mathbf{P}\left\{\sqrt{n}\left(\widetilde{L}_{n}-\mu_{\widetilde{L}_{n}}\right) / \sigma>x \pm 2 \delta\right\}=[1-\Phi(x \pm 2 \delta)](1+o(1)),
$$

uniformly with respect to $x$ such that $-B \leqslant x \pm 2 \delta \leqslant b_{n} n^{1 / 6}$. In particular, the relation (3.8) holds true uniformly in the range $-A \leqslant x \leqslant a_{n} n^{1 / 6}$. To prove (3.5), it remains to note that since $2 \delta a_{n} z_{n}=2 \sqrt{a_{n}} \rightarrow 0$, Lemma A.1 from Vandemaele and Veraverbeke [29] now yields

$$
1-\Phi(x \pm 2 \delta)=[1-\Phi(x)](1+o(1)),
$$

as $n \rightarrow \infty$, uniformly in the range $-A \leqslant x \leqslant a_{n} z_{n}$.

Pro of of (B.6). Let $I_{1}^{(j)}$ and $I_{2}^{(j)}$ denote the first and the second terms of $R_{n}^{(j)}$ (cf. (2.9) and (2.J0)), respectively, $j=1,2$. In this notation, $R_{n}=I_{1}^{(1)}-$ 
$I_{2}^{(1)}+I_{1}^{(2)}-I_{2}^{(2)}$ and

$$
\mathbf{P}\left\{\sqrt{n}\left|R_{n}\right| / \sigma>\delta\right\} \leqslant \sum_{k=1}^{2} \mathbf{P}\left\{\sqrt{n}\left|I_{k}^{(1)}\right| / \sigma>\delta / 4\right\}+\sum_{k=1}^{2} \mathbf{P}\left\{\sqrt{n}\left|I_{k}^{(2)}\right| / \sigma>\delta / 4\right\} .
$$

Thus, it suffices to show that for each positive $C$ (in particular, for $C=\sigma / 4$ ),

$$
\mathbf{P}\left\{\sqrt{n}\left|I_{k}^{(j)}\right|>C \delta\right\}=[1-\Phi(x)] o(1), \quad k, j=1,2,
$$

as $n \rightarrow \infty$, uniformly in the range $-A \leqslant x \leqslant a_{n} z_{n}$. We will prove (B. $\amalg$ ) for $I_{1}^{(1)}$ and $I_{1}^{(2)}$ (the treatment of $I_{2}^{(1)}$ and $I_{2}^{(2)}$ is similar, and therefore omitted).

Consider $I_{1}^{(1)}$. First, note that if $\alpha<A_{n}$, then $\max _{u \in\left(\alpha, A_{n}\right)}\left|F_{n}^{-1}(u)-\xi_{\alpha}\right|=$ $\xi_{\alpha}-X_{[n \alpha]+1: n} \leqslant \xi_{\alpha}-X_{[n \alpha]: n}$, as $F_{n}^{-1}$ is monotonic. Here and in what follows $[x]$ represents the greatest integer function. Similarly we find that if $A_{n} \leqslant \alpha$, then $\max _{u \in\left(A_{n}, \alpha\right)}\left|F_{n}^{-1}(u)-\xi_{\alpha}\right|=X_{[n \alpha]: n}-\xi_{\alpha}$. Moreover, by the Lipschitz condition for $J$, there exists a positive $K$ such that $\max _{u \in[0,1]} J_{w}(u) \leqslant \sup _{u \in I} J(u) \leqslant K$. This yields

$$
\left|I_{1}^{(1)}\right|=\left|\int_{\alpha}^{A_{n}} J_{w}(u)\left[F_{n}^{-1}(u)-\xi_{\alpha}\right] d u\right| \leqslant K\left|A_{n}-\alpha\right|\left|X_{[n \alpha]: n}-\xi_{\alpha}\right| .
$$

Define a sequence of intervals $\Gamma_{n}=\left[\alpha \wedge \alpha_{n}, \alpha \vee \alpha_{n}+1 / n\right)$; then we obtain

$$
\left|I_{1}^{(2)}\right|=\left|\int_{\alpha_{n}}^{\alpha} J(u)\left[F_{n}^{-1}(u)-F^{-1}(u)\right] d u\right| \leqslant K\left|\alpha_{n}-\alpha\right| D_{n},
$$

where $D_{n}=\max _{i: i / n \in \Gamma_{n}}\left|X_{i: n}-F^{-1}(i / n)\right| \vee\left|X_{i: n}-F^{-1}((i-1) / n)\right|$.

Let $U_{1}, \ldots, U_{n}$ be a sample of independent $(0,1)$-uniform distributed random variables, and $U_{i: n}$ the corresponding order statistics. Set $M_{\alpha}=\sharp\left\{i: U_{i} \leqslant \alpha\right\}$. Since the joint distribution of $X_{i: n}$ and $N_{\alpha}$ coincides with the joint distribution of $F^{-1}\left(U_{i: n}\right)$ and $M_{\alpha}, i=1, \ldots, n$, in order to prove (B.DI), it suffices to show that

$$
\begin{aligned}
\mathbf{P}\left\{\left|M_{\alpha}-n \alpha\right|\left|U_{[n \alpha]: n}-\alpha\right|^{\varepsilon}>C \sqrt{n} \delta\right\} & =[1-\Phi(x)] o(1), \\
\mathbf{P}\left\{\sqrt{n}\left|\alpha_{n}-\alpha\right| D_{n, u}^{\varepsilon}>C \delta\right\} & =[1-\Phi(x)] o(1), \\
\mathbf{P}\left(\bigcup_{i: i / n \in \Gamma_{n}}\left\{U_{i: n} \notin U_{\alpha}\right\}\right) & =[1-\Phi(x)] o(1),
\end{aligned}
$$

as $n \rightarrow \infty$, uniformly in the range $-A \leqslant x \leqslant a_{n} z_{n}$. Here $U_{\alpha}$ is the neighborhood of $\alpha$, in which $F^{-1}$ satisfies a Hölder condition of order $\varepsilon$ (cf. condition (ii)),

$$
D_{n, u}^{\varepsilon}=\max _{i: i / n \in \Gamma_{n}}\left|U_{i: n}-i / n\right|^{\varepsilon} \vee\left|U_{i: n}-(i-1) / n\right|^{\varepsilon},
$$


and $C$ stands for a positive constant independent of $n$, which may change its value from line to line.

To shorten the notation, let $k=[n \alpha]$. Consider the probability on the left-hand side in the first line of (3.14). It is equal to

$$
\mathbf{P}\left\{\left|M_{\alpha}-n \alpha\right|\left|U_{k: n}-\alpha\right|^{\varepsilon}>C a_{n}^{-1 / 2} n^{1 /(2+\varepsilon)}\right\} \leqslant \mathbf{P}_{1}+\mathbf{P}_{2},
$$

where

$$
\begin{aligned}
& \mathbf{P}_{1}:=\mathbf{P}\left\{\left|M_{\alpha}-n \alpha\right|>C_{1} a_{n}^{-1 /(2(1+\varepsilon))} n^{(1+\varepsilon) /(2+\varepsilon)}\right\}, \\
& \mathbf{P}_{2}:=\mathbf{P}\left\{\left|U_{k: n}-\alpha\right|^{\varepsilon}>C_{2} a_{n}^{-\varepsilon /(2(1+\varepsilon))} n^{-\varepsilon /(2+\varepsilon)}\right\},
\end{aligned}
$$

$C_{1}, C_{2}$ are any positive constants such that $C_{1} C_{2}=C$. Let us estimate $\mathbf{P}_{1}$ and $\mathbf{P}_{2}$. Set $h=C_{1} a_{n}^{-1 /(2(1+\varepsilon))} n^{(1+\varepsilon) /(2+\varepsilon)-1}$. Since $h<1-\alpha$ for all sufficiently large $n$ (because $a_{n} \geqslant 1 / \log (1+n)$ ), by Theorem 1 of Hoeffding [20] we have

(3.17)

$$
\mathbf{P}_{1}=\mathbf{P}\left\{\left|M_{\alpha}-n \alpha\right|>n h\right\} \leqslant 2 \exp \left(-2 n h^{2}\right)=2 \exp \left(-2 C_{1}^{2} n^{\varepsilon /(2+\varepsilon)} a_{n}^{-1 /(1+\varepsilon)}\right) .
$$

Next, we evaluate $1 /(1-\Phi(x))$. Let $\phi=\Phi^{\prime}$. Since $1-\Phi(x) \sim \phi(x) / x$ as $x \rightarrow \infty$, for $x$ such that $-A \leqslant x \leqslant a_{n} z_{n}$ we have

$\frac{1}{1-\Phi(x)} \leqslant \frac{1}{1-\Phi\left(a_{n} z_{n}\right)} \sim \frac{a_{n} z_{n}}{\phi\left(a_{n} z_{n}\right)}=\sqrt{2 \pi} a_{n} n^{\varepsilon /(2(2+\varepsilon))} \exp \left(a_{n}^{2} n^{\varepsilon /(2+\varepsilon)} / 2\right)$, and combining (3.17) and (3.18), we obtain

$$
\mathbf{P}_{1}=[1-\Phi(x)] o(1) \quad \text { as } n \rightarrow \infty,
$$

uniformly in the range $-A \leqslant x \leqslant a_{n} z_{n}$.

Set $p_{k}=k /(n+1)$, and note that $0<\alpha-p_{k}<n^{-1}$. Then for $\mathbf{P}_{2}$ we have

$$
\begin{aligned}
\mathbf{P}_{2} & \leqslant \mathbf{P}\left\{\left|U_{k: n}-p_{k}\right|>C_{2}^{1 / \varepsilon} a_{n}^{-1 /(2(1+\varepsilon))} n^{-1 /(2+\varepsilon)}-n^{-1}\right\} \\
& =\mathbf{P}\left\{\sqrt{n}\left|U_{k: n}-p_{k}\right|>C_{2}^{1 / \varepsilon} a_{n}^{-1 /(2(1+\varepsilon))} n^{\varepsilon /(2(2+\varepsilon))}-n^{-1 / 2}\right\} .
\end{aligned}
$$

Note that the term $n^{-1 / 2}$ on the right-hand side in (B.20) is of negligible order, and therefore we may omit it. Set $\lambda:=C_{2}^{1 / \varepsilon} a_{n}^{-1 /(2(1+\varepsilon))} n^{\varepsilon /(2(2+\varepsilon))}$. We observe that $\lambda / \sqrt{n}=C_{2}^{1 / \varepsilon} a_{n}^{-1 /(2(1+\varepsilon))} n^{-1 /(2+\varepsilon)}$, the latter quantity tends to zero because $a_{n} \geqslant 1 / \log (1+n)$, and so we can apply Inequality 1 and Proposition 1 (relation (12)) given on pages 453 and 455, respectively, in Shorack and Wellner [26]. Then we obtain

$$
\begin{aligned}
\mathbf{P}_{2} & \leqslant 2 \exp \left(-\frac{\lambda^{2}}{2 p_{k}} \frac{1}{1+2 \lambda /\left(3 p_{k} \sqrt{n}\right)}\right) \\
& =2 \exp \left(-\frac{1}{2 p_{k}} C_{2}^{2 / \varepsilon} a_{n}^{-1 /(1+\varepsilon)} n^{\varepsilon /(2+\varepsilon)}[1+o(1)]\right) .
\end{aligned}
$$


From (B.18) and (B.21) it follows that

$$
\mathbf{P}_{2}=[1-\Phi(x)] o(1) \quad \text { as } n \rightarrow \infty,
$$

uniformly in the range $-A \leqslant x \leqslant a_{n} z_{n}$. So, the first relation in (B.14) follows directly from (B.16), (B.19) and (B.22).

In the next step we prove the second relation in (3.14). We have

$$
\begin{aligned}
& \mathbf{P}\left\{\sqrt{n}\left|\alpha_{n}-\alpha\right| D_{n, u}^{\varepsilon}>C \delta\right\} \\
& \leqslant \sum_{i: i / n \in \Gamma_{n}} \mathbf{P}\left\{\sqrt{n}\left|\alpha_{n}-\alpha\right|\left|U_{i: n}-i / n\right|^{\varepsilon} \vee\left|U_{i: n}-(i-1) / n\right|^{\varepsilon}>C \delta\right\}
\end{aligned}
$$

By condition (iii), there exists $M>0$ such that $\left|\alpha_{n}-\alpha\right| \leqslant M n^{-1 /(2+\varepsilon)}$ for all sufficiently large $n$; hence each item of the sum on the right-hand side in (B.23) does not exceed

$$
\mathbf{P}\left\{\sqrt{n}\left|U_{i: n}-i / n\right|>\lambda\right\}+\mathbf{P}\left\{\sqrt{n}\left|U_{i: n}-(i-1) / n\right|>\lambda\right\}, \quad i / n \in \Gamma_{n},
$$

where $\lambda=C_{\varepsilon} a_{n}^{-1 /(2 \varepsilon)} n^{\varepsilon /(2(2+\varepsilon))}$ and $C_{\varepsilon}=(C / M)^{1 / \varepsilon}$. Obviously (cf. (B.20) and (B.21)), it suffices to prove the desired bound for the first of two probabilities in (3.24). Applying once more the exponential Inequality 1 for uniform order statistics (cf. Shorack and Wellner [26], pp. 453, 455) and the fact that $|i / n-\alpha| \leqslant$ $M n^{-1 /(2+\varepsilon)}$ for all sufficiently large $n$, we obtain

$$
\mathbf{P}\left\{\sqrt{n}\left|U_{i: n}-i / n\right|>\lambda\right\} \leqslant 2 \exp \left(-\frac{1}{2 \alpha} C_{\varepsilon}^{2} a_{n}^{-1 / \varepsilon} n^{\varepsilon /(2+\varepsilon)}\left[1+O\left(n^{-1 /(2+\varepsilon)}\right)\right]\right) .
$$

Since the number of items on the right-hand side in the relation (3.23) does not exceed $|n| \alpha-\alpha_{n}|+1|=O\left(n^{(1+\varepsilon) /(2+\varepsilon)}\right)$, the latter bound implies that the quantity on the right-hand side in (B.23) is of the order

$$
n^{(1+\varepsilon) /(2+\varepsilon)} \exp \left(-\frac{1}{2 \alpha} C_{\varepsilon}^{2} a_{n}^{-1 / \varepsilon} n^{\varepsilon /(2+\varepsilon)}[1+o(1)]\right) .
$$

This together with (B.18) implies the required relation.

Now, it remains to prove the last relation in (B.14). Fix some $\gamma>0$ such that $[\alpha-\gamma, \alpha+\gamma] \subseteq U_{\alpha}$, set $r_{n}=k \wedge k_{n}, s_{n}=k \vee k_{n}+1$, where $k_{n}=n \alpha_{n}$ (cf. (‥1)). Then

$$
\mathbf{P}\left(\bigcup_{i: i / n \in \Gamma_{n}}\left\{U_{i: n} \notin U_{\alpha}\right\}\right) \leqslant \mathbf{P}\left(U_{r_{n}: n}<\alpha-\gamma\right)+\mathbf{P}\left(U_{U_{s_{n}: n}: n}>\alpha+\gamma\right)
$$

Observe that both sequences $r_{n} / n$ and $s_{n} / n$ satisfy condition (iii), along with the sequence $\alpha_{n}=k_{n} / n$. Let us estimate the first probability on the right-hand side 
in (B.25]) (the treatment of the second one is similar). Define a binomial random variable $S_{n}=\sharp\left\{i: U_{i}<\alpha-\gamma\right\}$; then the first term on the right-hand side in (B.25) is equal to

$$
\begin{aligned}
\mathbf{P}\left(S_{n} \geqslant r_{n}\right) & =\mathbf{P}\left(S_{n}-\mathbf{E} S_{n} \geqslant r_{n}-n \alpha+\gamma n\right) \\
& =\mathbf{P}\left(n^{-1}\left(S_{n}-\mathbf{E} S_{n}\right) \geqslant \gamma+o(1)\right),
\end{aligned}
$$

and by the classical Hoeffding [20] inequality, the latter quantity is not greater than $\exp \left(-2 n(\gamma+o(1))^{2}\right)$, which is $[1-\Phi(x)] o(1)$, uniformly in the range $-A \leqslant x \leqslant a_{n} n^{1 / 2}$, and the last relation in (B.14) holds true.

The relations (B.12)-(B.14) directly imply (B.11), which yields (B.6)).

Pr o of of (B.7). By condition (iv), there exists $b>0$ such that

$$
\sqrt{n}\left|V_{n}\right| \leqslant b n^{-\varepsilon /(2(2+\varepsilon))}\left(\left|X_{\left(k_{n}+1\right): n}\right| \vee\left|X_{\left(n-m_{n}\right): n}\right|\right)
$$

for all sufficiently large $n$. Thus,

$$
\mathbf{P}\left(\sqrt{n}\left|V_{n}\right| / \sigma>\delta\right) \leqslant \mathbf{P}\left(\left|X_{\left(k_{n}+1\right): n}\right| \vee\left|X_{\left(n-m_{n}\right): n}\right|>\sigma a_{n}^{-1 / 2}\right) \leqslant \mathbf{P}_{3}+\mathbf{P}_{4},
$$

where $\mathbf{P}_{3}=\mathbf{P}\left(\left|X_{\left(k_{n}+1\right): n}\right|>\sigma a_{n}^{-1 / 2}\right), \mathbf{P}_{4}=\mathbf{P}\left(\left|X_{\left(n-m_{n}\right): n}\right|>\sigma a_{n}^{-1 / 2}\right)$. Let us estimate $\mathbf{P}_{3}$ (the treatment for $\mathbf{P}_{4}$ is the same, and therefore omitted). We have

$$
\begin{aligned}
\mathbf{P}_{3} & =\mathbf{P}\left(\left|F^{-1}\left(U_{\left(k_{n}+1\right): n}\right)\right|>\sigma a_{n}^{-1 / 2}\right) \\
& \leqslant \mathbf{P}\left(\left|F^{-1}\left(U_{\left(k_{n}+1\right): n}\right)-F^{-1}(\alpha)\right|+\left|F^{-1}(\alpha)\right|>\sigma a_{n}^{-1 / 2}\right) \\
& \leqslant \mathbf{P}\left(\left|U_{\left(k_{n}+1\right): n}-\alpha\right|^{\varepsilon}>\sigma a_{n}^{-1 / 2}(C+o(1))\right)+\mathbf{P}\left(U_{\left(k_{n}+1\right): n} \notin U_{\alpha}\right) .
\end{aligned}
$$

Observe that the first term on the right-hand side in (B.27) is equal to zero for all sufficiently large $n$, and the second one is $[1-\Phi(x)] o(1)$, uniformly in the range $-A \leqslant x \leqslant a_{n} z_{n}$. This completes the proof of (3.7) and the theorem.

Pro of of The ore m L.2. Let us first prove the relation (L.8). By Lemma 2.1 and the relation (B.2), we have

$$
\operatorname{Var}\left(L_{n}\right)=\operatorname{Var}\left(\widetilde{L}_{n}\right)+\operatorname{Var}\left(R_{n}+V_{n}\right)+2 \operatorname{cov}\left(\widetilde{L}_{n}, R_{n}+V_{n}\right) .
$$

Since $W_{i}$ are bounded, all conditions of Theorem 2 (ii) in [29] are satisfied, and hence

$$
\sigma^{-1} n^{1 / 2} \sqrt{\operatorname{Var}\left(\widetilde{L}_{n}\right)}=1+O\left(n^{-1 / 2}\right)
$$

(cf. [29], p. 431). Furthermore, we have

$$
\begin{aligned}
n\left|\operatorname{cov}\left(\widetilde{L}_{n}, R_{n}+V_{n}\right)\right| & \leqslant n\left[\operatorname{Var}\left(\widetilde{L}_{n}\right) \operatorname{Var}\left(R_{n}+V_{n}\right)\right]^{1 / 2} \\
& =\sigma\left[n \operatorname{Var}\left(R_{n}+V_{n}\right)\right]^{1 / 2}\left(1+O\left(n^{-1 / 2}\right)\right) .
\end{aligned}
$$


The latter three relations imply that in order to prove (‥8), it suffices to show that

$$
n \operatorname{Var}\left(R_{n}+V_{n}\right)=O\left(n^{-2 \varepsilon /(2+\varepsilon)}\right) .
$$

We have

$$
n \operatorname{Var}\left(R_{n}+V_{n}\right) \leqslant n \mathbf{E}\left(R_{n}+V_{n}\right)^{2} \leqslant 5 n\left[\sum_{i, j=1}^{2} \mathbf{E}\left(I_{j}^{(i)}\right)^{2}+\mathbf{E} V_{n}^{2}\right],
$$

where $I_{j}^{(i)}$ are as in (B.TO) and (B.Tl). We will show that

$$
n \mathbf{E}\left(I_{j}^{(1)}\right)^{2}=O\left(n^{-\varepsilon}\right)=o\left(n^{-2 \varepsilon /(2+\varepsilon)}\right), n \mathbf{E}\left(I_{j}^{(2)}\right)^{2}=O\left(n^{-2 \varepsilon /(2+\varepsilon)}\right), j=1,2,
$$

and that

$$
n \mathbf{E} V_{n}^{2}=O\left(n^{-2 \varepsilon /(2+\varepsilon)}\right) .
$$

The relations (B.29) -(B.3W) imply the desired bound (B.28).

We first prove (B.30), and consider in detail only the case $j=1$ (the treatment in the case $j=2$ is the same, and therefore omitted). Let, as before, $k=[\alpha n]$ and $k_{n}=\alpha_{n} n$. By (B.12) and the Schwarz inequality, we have

$$
\begin{aligned}
\mathbf{E}\left(I_{1}^{(1)}\right)^{2} & \leqslant K^{2}\left[\mathbf{E}\left(A_{n}-\alpha\right)^{4} \mathbf{E}\left(X_{k: n}-\xi_{\alpha}\right)^{4}\right]^{1 / 2} \\
& =K^{2} n^{-2}\left[\mathbf{E}\left(N_{\alpha}-\alpha n\right)^{4} \mathbf{E}\left(X_{k: n}-\xi_{\alpha}\right)^{4}\right]^{1 / 2} .
\end{aligned}
$$

By the well-known formula for the fourth moments of a binomial random variable, we have $\mathbf{E}\left(N_{\alpha}-\alpha n\right)^{4}=3 \alpha^{2}\left(1-\alpha^{2}\right) n^{2}(1+o(1))$. Thus, there exists a positive constant $C$ independent of $n$ such that

$$
n \mathbf{E}\left(I_{1}^{(1)}\right)^{2} \leqslant C\left[\mathbf{E}\left(X_{k: n}-\xi_{\alpha}\right)^{4}\right]^{1 / 2}
$$

for all sufficiently large $n$. We have

$$
\begin{aligned}
\mathbf{E}\left(X_{k: n}\right. & \left.-\xi_{\alpha}\right)^{4}=\mathbf{E}\left(F^{-1}\left(U_{k: n}\right)-F^{-1}(\alpha)\right)^{4} \\
= & \mathbf{E}\left[\left(F^{-1}\left(U_{k: n}\right)-F^{-1}(\alpha)\right)^{4} \mathbf{1}_{\left\{U_{k: n} \in U_{\alpha}\right\}}\right] \\
& +\mathbf{E}\left[\left(F^{-1}\left(U_{k: n}\right)-F^{-1}(\alpha)\right)^{4} \mathbf{1}_{\left\{U_{k: n} \notin U_{\alpha}\right\}}\right] \\
& \leqslant C_{H}^{4} \mathbf{E}\left|U_{k: n}-\alpha\right|^{4 \varepsilon}+\mathbf{E}\left[\left(X_{k: n}-\xi_{\alpha}\right)^{6}\right]^{2 / 3}\left[\mathbf{P}\left(U_{k: n} \notin U_{\alpha}\right)\right]^{1 / 3},
\end{aligned}
$$

where $C_{H}$ is a constant from the Hölder condition (ii). Note that if $\varepsilon>1 / 2$, then $\mathbf{E}\left|U_{k: n}-\alpha\right|^{4 \varepsilon} \leqslant \mathbf{E}\left|U_{k: n}-\alpha\right|^{2}=O\left(n^{-1}\right)$, and if $\varepsilon \leqslant 1 / 2$, then $\mathbf{E}\left|U_{k: n}-\alpha\right|^{4 \varepsilon} \leqslant$ $\left(\mathbf{E}\left|U_{k: n}-\alpha\right|^{2}\right)^{2 \varepsilon}=O\left(n^{-2 \varepsilon}\right)$. Since moments of any order of $X_{k: n}$ are finite for all sufficiently large $n$ and because $\mathbf{P}\left(U_{k: n} \notin U_{\alpha}\right)=O(\exp (-c n))$ with some $c>0$ 
(cf. (B.25), (B.26)), the latter bounds and the relations (B.32), (B.33) imply the first of the relations (B.30).

Consider $I_{1}^{(2)}$. By condition (iii'), there exists $d>0$ such that $\left(\alpha_{n}-\alpha\right)^{2} \leqslant$ $d n^{-\left(2+\varepsilon-\varepsilon^{2}\right) /(2+\varepsilon)}(\log n)^{-\varepsilon}$ for all sufficiently large $n$. Then, by (B.13), we obtain

$$
n \mathbf{E}\left(I_{1}^{(2)}\right)^{2} \leqslant n K^{2}\left(\alpha_{n}-\alpha\right)^{2} \mathbf{E} D_{n}^{2} \leqslant K^{2} n^{\varepsilon^{2} /(2+\varepsilon)}(\log n)^{-\varepsilon} \mathbf{E} D_{n}^{2} .
$$

Hence, to get the second bound in (3.30), it suffices to show that

$$
\mathbf{E} D_{n}^{2}=O\left((\log n)^{\varepsilon} n^{-\varepsilon}\right) .
$$

For all sufficiently large $n$, we have $\alpha_{n} \in U_{\alpha}$, and

$$
\mathbf{E} D_{n}^{2} \leqslant C_{H}^{2} \mathbf{E}\left(D_{n, u}^{\varepsilon}\right)^{2}=C_{H}^{2} \mathbf{E}\left(\max _{i: i / n \in \Gamma_{n}}\left|U_{i: n}-i / n\right|^{2 \varepsilon} \vee\left|U_{i: n}-(i-1) / n\right|^{2 \varepsilon}\right),
$$

where $D_{n}$ is as in (B.15)). The latter quantity does not exceed

$$
\begin{aligned}
& t^{\varepsilon} C_{H}^{2}(\log n)^{\varepsilon} n^{-\varepsilon} \\
& \quad+\mathbf{P}\left(\bigcup_{i: i / n \in \Gamma_{n}}\left\{\left|U_{i: n}-i / n\right| \vee\left|U_{i: n}-(i-1) / n\right|>\sqrt{t \frac{\log n}{n}}\right\}\right) \\
& \leqslant t^{\varepsilon} C_{H}^{2}(\log n)^{\varepsilon} n^{-\varepsilon}+\left|\alpha n-k_{n}+1\right|\left(\mathbf{P}_{1}+\mathbf{P}_{2}\right),
\end{aligned}
$$

where $t$ is a constant which will be chosen later, and

$\mathbf{P}_{1}=\mathbf{P}\left(\left|U_{i: n}-i / n\right|>\sqrt{t \frac{\log n}{n}}\right), \mathbf{P}_{2}=\mathbf{P}\left(\left|U_{i: n}-(i-1) / n\right|>\sqrt{t \frac{\log n}{n}}\right)$.

It is obvious that both $\mathbf{P}_{1}$ and $\mathbf{P}_{2}$ are of the same order of magnitude, so it suffices to estimate $\mathbf{P}_{1}$, where we can apply once more Inequality 1 from Shorack and Wellner [26]. We have

$$
\mathbf{P}_{1}=\mathbf{P}\left(\sqrt{n}\left|U_{i: n}-i / n\right|>\sqrt{t \log n}\right) \leqslant 2 \exp \left(-\frac{t \log n}{2 \alpha}\left(1+O\left(\left|\alpha_{n}-\alpha\right|\right)\right)\right) ;
$$

hence, if we choose $t \geqslant 4 \alpha$, we obtain $\mathbf{P}_{1}+\mathbf{P}_{2}=O\left(n^{-2}\right)$, and the second term on the right-hand side in (B.36) becomes negligible in order relative to the first one. This proves (B.35) and the second relation in (B.30).

We now turn to the proof of (B.3 II). By condition (iv'), there exists a constant $C>0$, not depending on $n$, such that $\sum_{i=k_{n}+1}^{n-m_{n}}\left|c_{i, n}-c_{i, n}^{0}\right| \leqslant C n^{(2-\varepsilon) /(2(2+\varepsilon))}$ for all sufficiently large $n$, and

$$
\begin{aligned}
n \mathbf{E} V_{n}^{2} & \leqslant n^{-1}\left(\sum_{i=k_{n}+1}^{n-m_{n}}\left|c_{i, n}-c_{i, n}^{0}\right|\right)^{2} \mathbf{E}\left(X_{k_{n}+1: n}^{2} \vee X_{n-m_{n}: n}^{2}\right) \\
& \leqslant C^{2} n^{-1} n^{(2-\varepsilon) /(2+\varepsilon)} \mathbf{E}\left(X_{k_{n}+1: n}^{2} \vee X_{n-m_{n}: n}^{2}\right)=O\left(n^{-2 \varepsilon /(2+\varepsilon)}\right),
\end{aligned}
$$

and (B.3I) holds true. 
Thus, the relation $(11.8)$ is proved, and we are now in a position to prove that the relations (‥7) hold true if we replace $\sigma / n^{1 / 2}$ by $\sqrt{\operatorname{Var}\left(L_{n}\right)}$. We prove the first of the relations $(\mathbb{L} .7)$, the second one will then follow from the first if we replace $c_{i, n}$ by $-c_{i, n}$.

Fix an arbitrary sequence $a_{n} \rightarrow 0$ and $A>0$, set $\lambda_{n}=\sigma^{-1} n^{1 / 2} \sqrt{\operatorname{Var}\left(L_{n}\right)}$, and write

$$
\frac{\mathbf{P}\left(\left(L_{n}-\mu_{n}\right) / \sqrt{\operatorname{Var}\left(L_{n}\right)}>x\right)}{1-\Phi(x)}=\frac{1-F_{L_{n}}\left(\lambda_{n} x\right)}{1-\Phi\left(\lambda_{n} x\right)} \frac{1-\Phi\left(\lambda_{n} x\right)}{1-\Phi(x)} .
$$

Set $B=A \sup _{n \in \mathbb{N}} \lambda_{n}$ and $b_{n}=\lambda_{n} a_{n}$. Since $\lambda_{n} \rightarrow 1$, the number $B$ exists and $b_{n} \rightarrow 0$. Hence, by Theorem L.], the first ratio on the right-hand side in (B.37) tends to one as $n \rightarrow \infty$, uniformly in $x$ such that $-B \leqslant \lambda_{n} x \leqslant b_{n} z_{n}$, where $z_{n}=$ $n^{\varepsilon /(2(2+\varepsilon))}$, in particular, uniformly in the range $-A \leqslant x \leqslant a_{n} z_{n}$. Furthermore, we see that $\left|\lambda_{n}-1\right|^{1 / 2} a_{n} z_{n} \rightarrow 0$, which is due to the fact that $\left|\lambda_{n}-1\right|^{1 / 2}=$ $O\left(n^{-\varepsilon /(2(2+\varepsilon))}\right)$. Hence, by Lemma A1 from Vandemaele and Veraverbeke [29], the second ratio on the right-hand side in (B.37) also tends to one, uniformly in the range $-A \leqslant x \leqslant a_{n} z_{n}$. Thus, the theorem is proved.

Acknowledgments. The author is grateful to the referee for his valuable remarks and suggestions that led to improvement of the article.

\section{REFERENCES}

[1] A. Aleskeviciene, Large and moderate deviations for L-statistics, Lithuanian Math. J. 31 (1991), pp. 145-156.

[2] V. Bentkus, F. Götze, and W. R. van Zwet, An Edgeworth expansion for symmetric statistics, Ann. Statist. 25 (1997), pp. 851-896.

[3] V. Bentkus and R. Zitikis, Probabilities of large deviations for L-statistics, Lithuanian Math. J. 30 (1990), pp. 215-222.

[4] S. Bjerve, Error bound for linear combinations of order statistics, Ann. Statist. 5 (1977), pp. 357-369.

[5] H. Boistard, Large deviations for L-statistics, Statist. Decisions 25 (2007), pp. 89-125.

[6] H. Callaert, M. Vandemaele, and N. Veraverbeke, A Cramér type large deviation theorem for trimmed linear combinations of order statistics, Comm. Statist. Theory Methods 11 (1982), pp. 2689-2698.

[7] H. A. David and H. N. Nagaraja, Order Statistics, third edition, Wiley, New York 2003.

[8] K. O. Friedrich, A Berry-Esseen bound for functions of independent random variables, Ann. Statist. 17 (1989), pp. 170-183.

[9] F. Gao and X. Zhao, Delta method in large deviations and moderate deviations for estimators, Ann. Statist. 39 (2011), pp. 1211-1240.

[10] F. Greselin, L. Madan, M. L. Puri, and R. Zitikis, L-functions, processes, and statistics in measuring economic inequality and actuarial risks, Stat. Interface 2 (2009), pp. 227 245.

[11] N. V. Gribkova, On analogues of Berry-Esseen inequality for truncated linear combinations of order statistics, Theory Probab. Appl. 38 (1993), pp. 142-149. 
[12] N. V. Gribkova and R. Helmers, The empirical Edgeworth expansion for a Studentized trimmed mean, Math. Methods Statist. 15 (2006), pp. 61-87.

[13] N. V. Gribkova and R. Helmers, On the Edgeworth expansion and the $M$ out of $N$ bootstrap accuracy for a Studentized trimmed mean, Math. Methods Statist. 16 (2007), pp. 142 176.

[14] N. V. Gribkova and R. Helmers, On a Bahadur-Kiefer representation of von Mises statistic type for intermediate sample quantiles, Probab. Math. Statist. 32 (2012), pp. 255-279.

[15] N. V. Gribkova and R. Helmers, Second order approximations for slightly trimmed means, Theory Probab. Appl. 58 (2014), pp. 383-412.

[16] R. Helmers, Edgeworth expansions for trimmed linear combinations of order statistics, in: Proceedings of the Second Prague Symposium on Asymptotic Statistics, P. Mandl and M. Hušková (Eds.), North-Holland, Amsterdam 1979, pp. 221-232.

[17] R. Helmers, Edgeworth expansions for linear combinations of order statistics with smooth weight functions, Ann. Statist. 8 (1980), pp. 1361-1374.

[18] R. Helmers, A Berry-Esseen theorem for linear combinations of order statistics, Ann. Probab. 9 (1981), pp. 342-347.

[19] R. Helmers, Edgeworth expansions for linear combinations of order statistics, Mathematical Centre Tracts, Vol. 105, Mathematisch Centrum, Amsterdam 1982.

[20] W. Hoeffding, Probability inequalities for sums of bounded random variables, J. Amer. Statist. Assoc. 58 (1963), pp. 13-30.

[21] D. M. Mason and G. K. Shorack, Necessary and sufficient conditions for asymptotic normality of trimmed L-statistics, J. Statist. Plan. Inference 25 (1990), pp. 111-139.

[22] H. Putter and W. R. van Zwet, Empirical Edgeworth expansions for symmetric statistics, Ann. Statist. 26 (1998), pp. 1540-1569.

[23] R. J. Serfling, Approximation Theorems of Mathematical Statistics, Wiley, New York 1980.

[24] G. R. Shorack, Asymptotic normality of linear combinations of functions of order statistics, Ann. Math. Statist. 40 (1969), pp. 2041-2050.

[25] G. R. Shorack, Functions of order statistics, Ann. Math. Statist. 43 (1972), pp. 412-427.

[26] G. R. Shorack and J. A. Wellner, Empirical Processes with Applications in Statistics, Wiley, New York 1986.

[27] S. M. Stigler, Linear functions of order statistics, Ann. Math. Statist. 40 (1969), pp. 770 788.

[28] S. M. Stigler, Linear functions of order statistics with smooth weight functions, Ann. Statist. 2 (1974), pp. 676-693.

[29] M. Vandemaele and N. Veraverbeke, Cramér type large deviations for linear combinations of order statistics, Ann. Probab. 10 (1982), pp. 423-434.

[30] A. W. van der Vaart, Asymptotic Statistics, Camb. Ser. Stat. Probab. Math., Vol. 3, Cambridge Univ. Press, Cambridge 1998.

[31] W. R. van Zwet, A Berry-Esseen bound for symmetric statistics, Z. Wahrsch. Verw. Gebiete 66 (1984), pp. 425-440.

Nadezhda Gribkova

St. Petersburg State University

Mathematics and Mechanics Faculty

199034, St. Petersburg

Universitetskaya nab. 7/9, Russia

E-mail: n.gribkova@spbu.ru

Received on 28.8.2015;

revised version on 28.2 .2016 